\title{
Diagnose oftmals verdeckter Depressionen beim Hausarzt vereinfachen
}

\author{
Der Forschungspreis 2012 des Kollegiums für Hausarztmedizin KHM geht an zwei \\ Studien mit Fokus auf den Dialog zwischen Arzt und Patient. Die Preisverleihung \\ fand am 21. Juni 2012 im Kultur- und Kongresszentrum Luzern im Rahmen der \\ 14. Fortbildungstagung des KHM statt.
}

Winfried Suske

1 Lombardo $\mathrm{P}$, Vaucher $\mathrm{P}$, Haftgoli N, Burnand B, Favrat B, Verdon F, Bischoff T, Herzig L. The ‘help» question doesn't help when screening for major depression: external validation of the three question screening test for primary care patients managed for physical complaints. BMC Medicine. 2011;9:114.

2 Whooley MA, Avins AL, Miranda J, Browner WS. Case-finding instruments for depression. Two questions are as good as many. J Gen Intern Med. 1997;12:439-445.

3 Arroll B, Goodyear-Smith F, Kerse N, Fishman T, Gunn J. Effect of the addition of a «help» question to two screening questions on specificity for diagnosis of depression in general practice: diagnostic validity study. BMJ. 2005; 331:884

4 Litschgi L. Der Arzt auf dem Sozius - tolerieren statt dirigieren. PrimaryCare. 2012;12:7-10.

Korrespondenz: Dr. Winfried Suske Healthworld (Schweiz) AG Sennweidstrasse 46 6312 Steinhausen

winfried.suske[at]healthworld.ch

\section{Hauptpreis}

Der mit 25000 Franken dotierte Hauptpreis des KHM geht an Patrick Lombardo und die von Dr. med. Lilli Herzig geleitete Forschungsgruppe des Instituts für Hausarztmedizin der Universität Lausanne (IUMG) für ihre Studie [1] zur Früherkennung von Depressionen beim Hausarzt.

«Wir haben diese hervorragende Studie, die von Hausärzten in Zusammenarbeit mit einem universitären Zentrum konzipiert und realisiert wurde, aus dreizehn Forschungsprojekten ausgewählt, weil sie für alle Ärzte einen direkten Praxisbezug hat. Depressionen sind beim Hausarzt häufig anzutreffen, aber schwierig zu diagnostizieren. Meist sind sie durch unerklärliche andere somatische Symptome verdeckt, welche in der Regel der einzige Grund für die Konsultation sind», erklärt der Jury-Präsident Hans Stalder, Honorarprofessor der Universität Genf.

\section{Zentrale Rolle des Hausarztes}

Im Rahmen der zwischen November 2004 und Juli 2005 in der Westschweiz unter Mitarbeit von 24 Hausärzten durchgeführten Studie stellten die Ärzte den teilnehmenden Patienten zwei einfache Fragen (ursprünglich von Whooley et al. [2] vorgeschlagen) zur Erkennung von Depressionen: 1. «Waren Sie im letzten Monat häufig traurig, deprimiert, verzweifelt?»; 2. «Hatten Sie letzten Monat weniger Interesse und Lust an den meisten Aktivitäten, die Sie sonst gerne ausüben?»

Das Studienprotokoll wurde durch eine dritte Frage, die sogenannte «help»-Frage ergänzt: «Wünschen Sie diesbezüglich Hilfe?», welche in etwa zur selben Zeit von Arroll et al. [3] zur Erkennung von Depressionen vorgeschlagen wurde.

9 von 10 Depressionen mittels zwei Fragen erkannt Die Auswertung der Antworten der 724 Patienten, die eingewilligt hatten zu Beginn und Schluss der Studie alle drei Fragen zu beantworten, zeigt, dass die ersten zwei Fragen ausreichen, um in neun von zehn Fällen eine schwere Depression zu erkennen. Wird mindes- tens eine der beiden Fragen bejaht, wird der Test positiv gewertet. Die Studie hat bei 9,5\% der Patienten, die ihren Hausarzt wegen somatischer Beschwerden aufsuchten, eine schwere Depression festgestellt.

Die «help»-Frage hingegen reduziert die Sensibilität der Früherkennung: Vier von zehn schwer depressiven Patienten geben an, keine Hilfe zu benötigen. Deshalb sollte die «help»-Frage nicht ins Früherkennungs-Tool aufgenommen werden. Sie kann aber im weiteren Verlauf von Bedeutung sein, wenn die Diagnose gestellt ist und es darum geht, jene Patienten ausfindig zu machen, die an einer Behandlung ihrer Depression interessiert sind.

\section{Anerkennungspreis}

Die Jury hat Dr. med. Louis Litschgi, Allgemeinmediziner in Basel, einen Anerkennungspreis verliehen für seine originelle Studie zur Behandlung von Patienten, welche die von ihrem Arzt vorgeschlagene Therapie ablehnen [4]. Er ging dabei folgender Frage nach: «Was geschieht, wenn sich der Patient bewusst für eine Behandlung entscheidet, die nicht den aktuellen medizinischen Erkenntnissen entspricht?» Zur Beantwortung dieser Fragen verfolgte Dr. Litschgi mit einigen seiner Kollegen 80 Fälle während sechs Jahren.

Im Rahmen einer «partizipativen» Medizin überliessen die Ärzte dem Patienten die Richtungswahl, unterstützten ihn aber bei seinen Therapie-Entscheidungen, auch wenn diese aus medizinischer Sicht riskant schienen. Die Resultate sprechen für sich: Bei 27\% der Patienten verlief die Genesung besser, als sie mit einer konventionellen Behandlung zu erwarten gewesen wäre. Nur bei 14\% der Patienten war sie weniger zufriedenstellend. Ausserdem fielen die Gesundheitskosten in zwei Dritteln der Fälle weniger hoch aus, und sowohl die Ärzte als auch die an der Studie teilnehmenden Patienten waren insgesamt sehr zufrieden.

Ausführliche Berichte zu den KHM-Preisen 2012 finden Sie in der aktuellen Ausgabe von PrimaryCare (Nr. 13/ 2012) unter www.primary-care.ch 